\title{
CROSS-SECTIONAL STUDY OF NEED OF TARGETED USG AND ITS BENEFITS IN DECREASING FOETAL AND MATERNAL MORBIDITY AND MORTALITY BY OBSTETRIC DECISION MAKING IN A LOW RESOURCE SETTING
}

\author{
Udaya Aruna Sundaram¹, Uma Dharmalingam², Sudharshini Thayuman Santhakumar ${ }^{3}$ \\ ${ }^{1}$ Assistant Professor, Department of Obstetrics and Gynaecology, KAPVGMC, MGMGH, Trichy. \\ ${ }^{2}$ Professor, Department of Obstetrics and Gynaecology, KAPVGMC, MGMGH, Trichy. \\ 3Junior Resident, Department of Obstetrics and Gynaecology, KAPVGMC, MGMGH, Trichy.
}

\begin{abstract}
\section{BACKGROUND}

Congenital anomalies are responsible for a remarkable proportion of mortality and morbidity in newborns. This paper was focused on incidence of structural congenital malformations detectable at birth among 6380 deliveries. This study was conducted to evaluate the need for targeted USG and its benefits in decreasing foetal and maternal morbidity and mortality by obstetric decision making in a low resource setting by assessing structural congenital anomalies and to predict the variables which coexist in the incidence of congenital anomalies so that we can reduce the related perinatal morbidity and mortality.
\end{abstract}

\section{MATERIALS AND METHODS}

This is a cross-sectional study carried out in the Dept. of OB-GYN at Mahatma Gandhi Memorial Government Hospital attached to KAPV Government Medical College, Trichy during 10 months period from April 2016 to January 2017. A total of 6380 deliveries over 10 months, from April 2016 to January 2017, were studied for gross congenital malformations at birth as well as diagnosed antenatally by USG. All congenital anomaly cases detected at delivery were included in the study and also data from the birth register, and available medical \& USG records during antenatal period were studied.

\section{RESULTS}

We diagnosed 104 cases of foetal anomalies. The antenatal prevalence of congenital anomalies was $1.63 \%$. Out Of 6380 deliveries, 104 cases were identified with a primary diagnosis of congenital anomalies giving a prevalence rate of $1.63 \%$. Anomalies of the cardiovascular system were the most common defects; accounting for $40.38 \%$ of birth defects followed by CNS anomalies of $25.0 \%$ \& GIT anomalies of $18.27 \%$. The surgery rate for delivery for anomaly group $19-22$ weeks cases was $11.77 \%$, which is very low when compared to $23-40$ weeks anomaly group cases, where the surgery rate was $42.53 \%$. Perinatal morbidity rate is $105.77 \%$ and mortality rate is $894.23 \%$ in anomaly group which is higher than the normal foetus group where morbidity rate is only $1.59 \%$ and mortality rate is $3.60 \%$.

\section{CONCLUSION}

The targeted USG and followup, decision making by specialists is important in reducing foetal and also maternal morbidity and mortality. Prevalence of congenital anomaly was $1.63 \%$ and anomalies of the cardiovascular system were the most common observed in our study. Majority cases were not associated with any risk factor. However, GDM is the most commonly associated one. Others are polyhydramnios and IUGR. Routine targeted USG by radiologist at 18-22 weeks is an important measure for early detection of malformation. This helps in primary prevention of disability and reduces perinatal morbidity and mortality. This prenatal diagnosis helps in reducing surgery rate and reduces maternal morbidity and mortality.

\section{KEYWORDS}

Congenital Anomaly, Targeted USG.

HOW TO CITE THIS ARTICLE: Sundaram UA, Dharmalingam U, Santhakumar ST. Cross-sectional study of need of targeted USG and its benefits in decreasing foetal and maternal morbidity and mortality by obstetric decision making in a low resource setting. J. Evolution Med. Dent. Sci. 2017;6(57):4236-4241, DOI: 10.14260/Jemds/2017/919

\section{BACKGROUND \\ Every year, about 7.9 million infants $(6 \%$ of worldwide births) are born with serious birth defects. With the causes of over $50 \%$ of birth defects unknown, how do we diagnose and prevent them?}

Financial or Other, Competing Interest: None.

Submission 15-04-2017, Peer Review 04-07-2017,

Acceptance 10-07-2017, Published 17-07-2017.

Corresponding Author:

Dr. Uma Dharmalingam,

\#19/23, South Ramalinga Nagar

Vayaloor Road,

Trichy-620017.

E-mail:ogdrdu@gmail.com

DOI: $10.14260 /$ jemds $/ 2017 / 919$
Congenital anomalies are a global health problem. Every year an estimated 7.9 million children are born with a serious birth defect, 3.3 million children (Under five years) die from birth defects, and 3.2 million who survive may develop a disability later in the life.1,2 They are the leading causes of prenatal mortality and childhood morbidity and disability in many countries.

\footnotetext{
Aims and Objectives

To evaluate the need for targeted USG at 18-22 weeks and followup and its benefits in decreasing foetal and maternal morbidity and mortality by obstetric decision making in a low resource setting [by assessing structural congenital anomalies that are diagnosed prenatally \& postnatally and by predicting the variables which coexist in the incidence of congenital anomalies].
} 


\section{MATERIALS AND METHODS}

The diagnosis of congenital abnormality was based on clinical evaluation and ultrasound examination (as documented by doctors in the patients' folders). Patient's history, including antenatal history, history of exposure to teratogens and family history of consanguinity were obtained from these folders. Further information obtained include maternal age, gestational age and type of congenital abnormality.

\section{Inclusion Criteria}

For inclusion in the study, a live birth must have been born from April 2016 to January 2017. Total prevalence was calculated by dividing the numerator (registered cases of congenital anomalies) by the relevant denominator (total live births) for the same period of time. Cases included are-

1. All deliveries which occurred at MGMGH, Trichy.

2. From review of all the records of babies born in MGMGH, Trichy admitted in the Newborn Intensive Care Unit, Mahatma Gandhi Memorial Hospital with the diagnosis of congenital abnormality.

A child with more than one anomaly was counted once only based on the primary diagnosis. We calculated $95 \%$ confidence interval (CI) for each prevalence rate.

For purposes of this study, congenital abnormalities are defined as obvious abnormality of structure or form which is present at birth or noticed within a few days after birth.3,4
Statistical Analysis: The study was analysed by the number and percentage for each variable mentioned in the tables.

\section{RESULTS}

We diagnosed 104 cases of foetal anomalies. The antenatal prevalence of congenital anomalies was $1.63 \%$. Out Of 6380 deliveries, 104 cases were identified with a primary diagnosis of congenital anomalies giving a prevalence rate of $1.63 \%$. Anomalies of the cardiovascular system were the most common defects, accounting for $40.38 \%$ of birth defects followed by CNS anomalies of $25.0 \%$ \& GIT anomalies of $18.27 \% .^{5}$ The surgery rate for delivery for anomaly group 19 22 weeks cases was $11.77 \%$, which is very low when compared to 23-40 weeks anomaly group cases, where the surgery rate was $42.53 \%$. Perinatal morbidity rate is $105.77 \%$ and mortality rate is $894.23 \%$ in anomaly group which is higher than the normal foetus group where morbidity rate is only $1.59 \%$ and mortality rate is $3.60 \%$. In the anomaly group of $19-22$ weeks, $88.23 \%$ of them were delivered vaginally and there is no foetal morbidity.

\begin{tabular}{|c|c|c|c|c|c|c|c|c|c|c|c|}
\hline \multirow{4}{*}{ Sl. No. } & \multirow{4}{*}{ Systems } & \multicolumn{8}{|c|}{ Congenital Abnormalities } & \multirow{4}{*}{ No. } & \multirow{4}{*}{$\%$} \\
\hline & & \multicolumn{4}{|c|}{ 19-22 wks. } & \multicolumn{4}{|c|}{ 23-40 wks. } & & \\
\hline & & \multicolumn{2}{|c|}{ Lethal } & \multicolumn{2}{|c|}{ Nonlethal } & \multicolumn{2}{|c|}{ Lethal } & \multicolumn{2}{|c|}{ Nonlethal } & & \\
\hline & & Type & No. & Type & No. & Type & No. & Type & No. & & \\
\hline 1. & CVS & & & & & $\begin{array}{l}\text { ASD } \\
\text { VSD } \\
\text { PDA- } \\
\text { PS } \\
\text { SV }\end{array}$ & $\begin{array}{c}8 \\
6 \\
25 \\
2 \\
1\end{array}$ & & & 42 & $40.38 \%$ \\
\hline 2. & CNS & $\begin{array}{l}\text { Hydrocephalus } \\
\text { Anencephalus } \\
\text { Encephalocoele }\end{array}$ & $\begin{array}{l}3 \\
5 \\
4\end{array}$ & & & $\begin{array}{l}\text { Myelomeningocoele } \\
\text { Hydrocephalus } \\
\text { Microcephaly } \\
\text { Spina bifida }\end{array}$ & $\begin{array}{l}6 \\
3 \\
2 \\
3\end{array}$ & & & 26 & $25 \%$ \\
\hline 3. & GIT & $\mathrm{CDH}$ & 2 & & & $\begin{array}{c}\text { Duodenal atresia } \\
\text { Oesophageal atresia } \\
\text { TEF } \\
\text { CDH } \\
\text { Ileal atresia }\end{array}$ & $\begin{array}{l}4 \\
1 \\
2 \\
2 \\
1\end{array}$ & $\begin{array}{c}\text { Cleft lip } \\
\text { Cleft palate }\end{array}$ & $\begin{array}{l}5 \\
2\end{array}$ & 19 & $18.27 \%$ \\
\hline 4. & Renal & & & $\begin{array}{c}\text { Post- } \\
\text { urethral } \\
\text { valve }\end{array}$ & 2 & Polycystic kidney & 3 & $\begin{array}{l}\text { Post- } \\
\text { urethral } \\
\text { valve }\end{array}$ & 1 & 6 & $5.77 \%$ \\
\hline 5. & Others & Multiple anomalies & 1 & & & $\begin{array}{l}\text { Down syndrome } \\
\text { Choanal atresia } \\
\text { Multiple anomalies } \\
\text { Anophthalmia }\end{array}$ & $\begin{array}{l}2 \\
1 \\
4 \\
1\end{array}$ & $\begin{array}{c}\text { Talipes } \\
\text { equinovarus }\end{array}$ & 2 & 11 & $10.58 \%$ \\
\hline
\end{tabular}




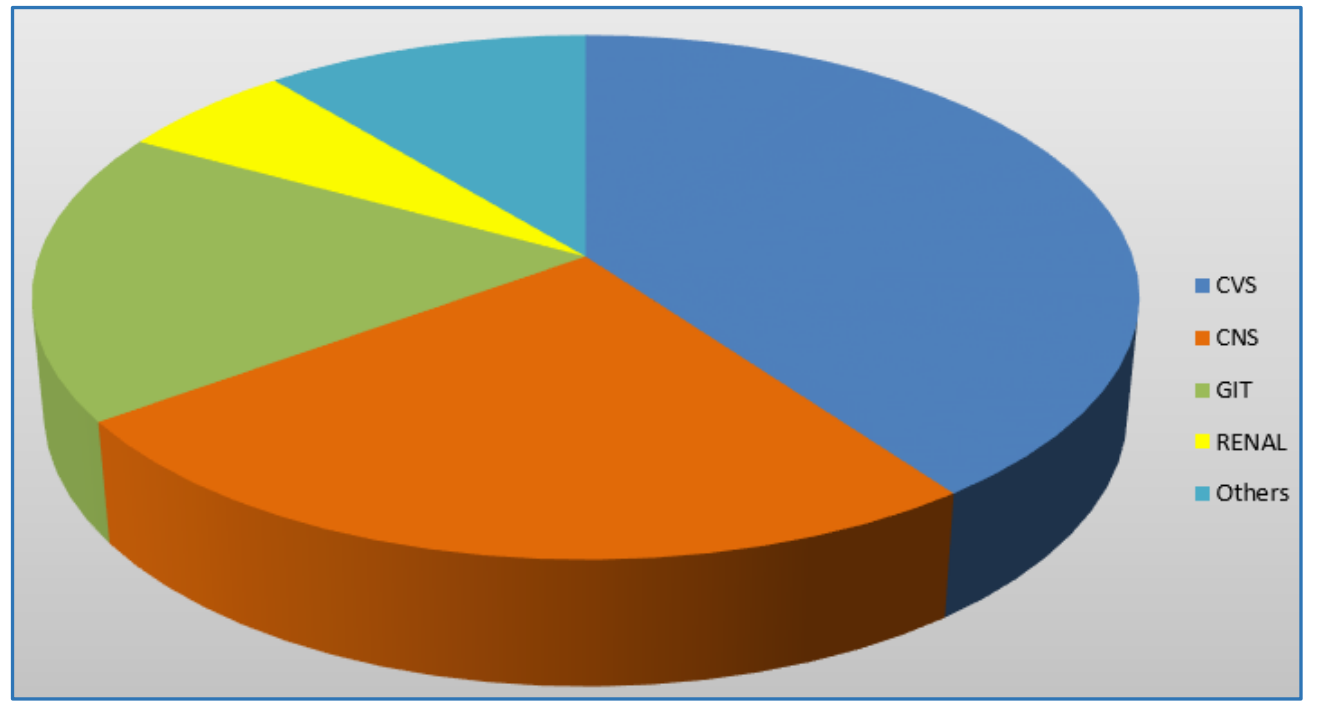

\begin{tabular}{|c|c|c|c|c|c|c|c|c|c|c|c|c|}
\hline \multirow{3}{*}{ Liquor Volume } & \multicolumn{10}{|c|}{ Systems } & \multirow{3}{*}{ No. } & \multirow{3}{*}{$\%$} \\
\hline & \multicolumn{2}{|c|}{ CVS } & \multicolumn{2}{|l|}{ CNS } & \multicolumn{2}{|l|}{ GIT } & \multicolumn{2}{|l|}{ Renal } & \multicolumn{2}{|l|}{ Others } & & \\
\hline & Type & No. & Type & No. & Type & No. & type & No. & Type & No. & & \\
\hline $\begin{array}{l}\text { Polyhydramnios } \\
\text { (AFI }>8 \text { ) }\end{array}$ & $\begin{array}{l}\text { ASD } \\
\text { VSD } \\
\text { PDA }\end{array}$ & $\begin{array}{l}2 \\
3 \\
5\end{array}$ & $\begin{array}{c}\text { Myelomeningocoel } \\
\text { e } \\
\text { Hydrocephalus- } \\
\text { Anencephaly } \\
\end{array}$ & $\begin{array}{l}6 \\
4 \\
5\end{array}$ & $\begin{array}{c}\text { Oeso-atresia } \\
\text { TEF } \\
\text { Cleft Palate- }\end{array}$ & $\begin{array}{l}1 \\
2 \\
2\end{array}$ & & & $\begin{array}{l}\text { Multiple } \\
\text { Anomaly }\end{array}$ & 1 & 31 & $29.81 \%$ \\
\hline $\begin{array}{l}\text { Oligohydramnios } \\
\text { (AFI<6) }\end{array}$ & $\begin{array}{l}\text { ASD } \\
\text { VSD } \\
\text { PDA } \\
\text { PS } \\
\text { Sv } \\
\end{array}$ & $\begin{array}{l}1 \\
1 \\
1 \\
2 \\
1 \\
\end{array}$ & & & & & $\begin{array}{l}\text { Polycystic } \\
\text { Kidney }\end{array}$ & 2 & $\begin{array}{l}\text { Multiple } \\
\text { Anomaly }\end{array}$ & 2 & 10 & $9.62 \%$ \\
\hline $\begin{array}{l}\text { Normal Liquor } \\
\quad \text { (AFI 6-8) }\end{array}$ & $\begin{array}{l}\text { ASD } \\
\text { VSD } \\
\text { PDA }\end{array}$ & $\begin{array}{c}5 \\
2 \\
19\end{array}$ & $\begin{array}{l}\text { Hydrocephalus } \\
\text { Microcephaly } \\
\text { Spina bifida, } \\
\text { Encephalocoele }\end{array}$ & $\begin{array}{l}2 \\
2 \\
3 \\
4\end{array}$ & $\begin{array}{c}\text { Duodenal atresia } \\
\text { CDH } \\
\text { Ileal Atresia, } \\
\text { Cleft Lip }\end{array}$ & $\begin{array}{l}4 \\
4 \\
5 \\
1\end{array}$ & 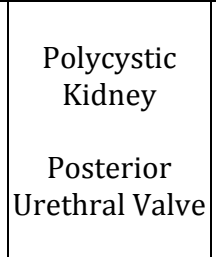 & $\begin{array}{l}1 \\
3\end{array}$ & \begin{tabular}{|c|} 
Down \\
Syndrome- \\
Choanal Atresia \\
-Talipes- \\
Anophthalmia- \\
Multiple \\
Anomaly
\end{tabular} & $\begin{array}{l}2 \\
1 \\
2 \\
1 \\
2\end{array}$ & 63 & $60.58 \%$ \\
\hline
\end{tabular}

As per Table 2 liquor volume has no significant correlation with the incidence of anomalies and $60.58 \%$ of anomalies were with normal amount of liquor.

Recommendations: 1. For polyhydramnios- karyotyping, biochemical testing, amnioreduction. 2. For oligohydramnioscounselling for prognosis, vesicoamniotic shunt in foetal obstructive uropathy, amnioinfusion. 3. For normal liquor- followup USG.

\begin{tabular}{|c|c|c|c|c|c|c|c|c|c|c|c|c|}
\hline \multirow{3}{*}{$\begin{array}{c}\text { Baby } \\
\text { Weight }\end{array}$} & \multicolumn{10}{|c|}{ System } & \multirow{3}{*}{ No. } & \multirow{3}{*}{$\%$} \\
\hline & \multicolumn{2}{|c|}{ CVS } & \multicolumn{2}{|l|}{ CNS } & \multicolumn{2}{|c|}{ GIT } & \multicolumn{2}{|l|}{ Renal } & \multicolumn{2}{|l|}{ Others } & & \\
\hline & Type & No. & Type & No. & Type & & Type & No. & Type & No. & & \\
\hline $\begin{array}{l}\text { Below } \\
2.6 \mathrm{~kg}\end{array}$ & $\begin{array}{l}\text { ASD } \\
\text { VSD } \\
\text { PDA } \\
\text { PS } \\
\text { SV }\end{array}$ & $\begin{array}{c}4 \\
3 \\
13 \\
2 \\
1\end{array}$ & $\begin{array}{l}\text { Myelomeningocoele } \\
\text { Hydrocephalus } \\
\text { Anencephalus }\end{array}$ & $\begin{array}{l}6 \\
6 \\
5\end{array}$ & $\begin{array}{c}\text { Oeso-atresia } \\
\text { TEF } \\
\text { CDH } \\
\text { Ileal Atresia }\end{array}$ & $\begin{array}{l}1 \\
2 \\
4 \\
1\end{array}$ & $\begin{array}{c}\text { Polycystic } \\
\text { Kidney }\end{array}$ & 3 & $\begin{array}{c}\text { Multiple } \\
\text { Anomalies }\end{array}$ & 4 & 55 & $52.88 \%$ \\
\hline $\begin{array}{c}2.6 \\
\text { Kg } \\
\text { \& Above }\end{array}$ & $\begin{array}{l}\text { ASD } \\
\text { VSD } \\
\text { PDA }\end{array}$ & $\begin{array}{c}4 \\
3 \\
12\end{array}$ & $\begin{array}{l}\text { Microcephaly } \\
\text { Spina Bifida } \\
\text { Encephalocoele }\end{array}$ & $\begin{array}{l}2 \\
3 \\
4\end{array}$ & $\begin{array}{l}\text { Duodenal } \\
\text { atresia } \\
\text { cleft lip } \\
\text { cleft palate }\end{array}$ & $\begin{array}{l}5 \\
2\end{array}$ & $\begin{array}{l}\text { Post- } \\
\text { Urethral } \\
\text { Valve }\end{array}$ & 3 & $\begin{array}{c}\text { Down Syndrome } \\
\text { Choanal Atresia } \\
\text { Talipes } \\
\text { Anophthalmia } \\
\text { Multiple } \\
\text { Anomalies }\end{array}$ & $\begin{array}{l}2 \\
1 \\
2 \\
1 \\
1\end{array}$ & 49 & $47.12 \%$ \\
\hline
\end{tabular}

Table 3 shows congenital anomalies have no significant correlation with baby weight.

Recommendations: 1 . For babies $2.5 \mathrm{~kg} \&$ below- genetic karyotyping, followup USG, induction based on karyotyping, USG abnormality \& gestational age.6,7 2 . For babies $2.6 \mathrm{~kg} \&$ above- followup USG, induction based on liquor volume \& gestational age. 


\begin{tabular}{|c|c|c|c|c|c|c|c|c|c|c|c|c|}
\hline \multicolumn{13}{|c|}{ GDM } \\
\hline & \multicolumn{10}{|c|}{ System } & \multirow{3}{*}{ No. } & \multirow{3}{*}{$\%$} \\
\hline & \multicolumn{2}{|c|}{ CVS- } & \multicolumn{2}{|l|}{ CNS- } & \multicolumn{2}{|l|}{ GIT } & \multicolumn{2}{|l|}{ Renal } & \multicolumn{2}{|l|}{ Others } & & \\
\hline & Type & No. & Type & No. & Type & No. & Type & No. & Type & No. & & \\
\hline $\begin{array}{l}\text { With } \\
\text { GDM }\end{array}$ & $\begin{array}{l}\text { ASD } \\
\text { VSD } \\
\text { PDA } \\
\text { PS } \\
\text { SV }\end{array}$ & $\begin{array}{c}6 \\
4 \\
15 \\
2 \\
1\end{array}$ & $\begin{array}{c}\text { Myelomeningocoele } \\
\text { Hydrocephalus, } \\
\text { Microcephaly } \\
\text { Spina bifida } \\
\text { Anencephaly } \\
\text { Encephalocoele } \\
\end{array}$ & $\begin{array}{l}5 \\
4 \\
2 \\
3 \\
3 \\
3\end{array}$ & & & $\begin{array}{c}\text { Polycystic } \\
\text { Kidney }\end{array}$ & 1 & $\begin{array}{l}\text { Multiple } \\
\text { Anomaly }\end{array}$ & 1 & 50 & $48.08 \%$ \\
\hline $\begin{array}{c}\text { Without } \\
\text { GDM }\end{array}$ & $\begin{array}{l}\text { ASD } \\
\text { VSD } \\
\text { PDA }\end{array}$ & $\begin{array}{c}2 \\
\\
2 \\
10\end{array}$ & $\begin{array}{l}\text { Myelomeningocoele } \\
\text { Hydrocephalus } \\
\text { Anencephaly } \\
\text { Encephalocoele }\end{array}$ & $\begin{array}{l}1 \\
2 \\
2 \\
1\end{array}$ & $\begin{array}{c}\text { Duodenal Atresia } \\
\text { Oeso-atresia } \\
\text { TEF } \\
\text { CDH } \\
\text { ILEAL Atresia } \\
\text { Cleft Lip } \\
\text { Cleft Palate }\end{array}$ & $\begin{array}{l}4 \\
1 \\
2 \\
4 \\
1 \\
5 \\
2 \\
\end{array}$ & $\begin{array}{l}\text { Polycystic } \\
\text { Kidney } \\
\text { Post- } \\
\text { urethral } \\
\text { Valve }\end{array}$ & $\begin{array}{l}2 \\
3\end{array}$ & $\begin{array}{c}\text { Down } \\
\text { Syndrome- } \\
\text { Choanal Atresia } \\
\text { Talipes } \\
\text { Anophthalmia } \\
\text { Multiple } \\
\text { Anomaly } \\
\end{array}$ & $\begin{array}{l}2 \\
1 \\
2 \\
1 \\
4 \\
4\end{array}$ & 54 & $51.92 \%$ \\
\hline
\end{tabular}

Table 4 shows GDM alone is not a risk factor for anomalies.

Recommendations: 1 . With GDM- Repeat biochemical testing, preconceptional counselling, control of metabolic state, targeted USG \& detailed evaluation. 2. Without GDMDecision based on type of anomaly. ${ }^{8}$

\section{Counselling has been given}

For cases diagnosed: 1. For lethal anomalies- termination. 2. For nonlethal anomalies- followup USG, on care pathway \& obstetric decision making. 3. For all cases diagnosed- about chances of recurrences, preconception control of metabolic state and necessity for targeted USG examination.

\begin{tabular}{|c|c|c|c|c|c|c|}
\hline \multicolumn{7}{|c|}{ Mode of Delivery-Anomaly Group } \\
\hline $\begin{array}{c}\text { Gestational } \\
\text { Age at } \\
\text { Diagnosis- } \\
\text { Weeks }\end{array}$ & No. & $\%$ & $\begin{array}{c}\text { Vaginal } \\
\text { Delivery } \\
\text { No. }\end{array}$ & $\%$ & $\begin{array}{c}\text { LSCS } \\
\text { No. }\end{array}$ & $\%$ \\
\hline $19-22$ & 17 & 16.35 & 15 & 88.23 & 2 & 11.77 \\
\hline $23-26$ & 4 & 3.85 & - & - & 4 & 100 \\
\hline $27-30$ & 4 & 3.85 & - & - & 4 & 100 \\
\hline $31-34$ & 20 & 19.23 & 14 & 70 & 6 & 30 \\
\hline $35-38$ & 30 & 28.85 & 21 & 70 & 9 & 30 \\
\hline $39 \&$ Above & 29 & 27.89 & 15 & 51.72 & 14 & 48.28 \\
\hline Total & $\mathbf{1 0 4}$ & $\mathbf{1 0 0}$ & $\mathbf{6 5}$ & $\mathbf{6 2 . 5}$ & $\mathbf{3 9}$ & $\mathbf{3 7 . 5}$ \\
\hline \multicolumn{7}{|c|}{ Table 5. Mode of Delivery } \\
\hline
\end{tabular}

\begin{tabular}{|c|c|c|c|c|c|c|}
\hline \multicolumn{6}{|c|}{ Mode of Delivery- Anomaly Group 23-40 Weeks } \\
\hline $\begin{array}{c}\text { Gest. } \\
\begin{array}{c}\text { Age- } \\
\text { wks. }\end{array}\end{array}$ & No. & $\%$ & Vaginal Delivery & \multicolumn{2}{c|}{ LSCS } \\
\cline { 4 - 7 } & & & No. & $\%$ & \multicolumn{2}{|c|}{ No. } \\
\hline $23-40$ wks. & 87 & 83.65 & 50 & 57.47 & 37 & 42.53 \\
\hline
\end{tabular}

\begin{tabular}{|c|c|c|c|c|c|c|}
\hline \multicolumn{7}{|c|}{ Mode of Delivery- Normal Foetus Group } \\
\hline $\begin{array}{c}\text { Gestational } \\
\text { age-Weeks }\end{array}$ & No. & $\mathbf{\%}$ & $\begin{array}{c}\text { Vaginal } \\
\text { Delivery-No. }\end{array}$ & $\mathbf{\%}$ & $\begin{array}{c}\text { LSCS } \\
\text { No. }\end{array}$ & $\mathbf{\%}$ \\
\hline $19-22$ & 840 & 13.38 & 825 & 98.21 & 15 & 1.79 \\
\hline $23-26$ & 1010 & 16.09 & 598 & 59.21 & 412 & 40.79 \\
\hline $27-30$ & 1216 & 19.38 & 604 & 49.67 & 612 & 50.33 \\
\hline $31-34$ & 1412 & 22.49 & 709 & 50.21 & 703 & 49.79 \\
\hline $35-38$ & 980 & 15.63 & 314 & 32.04 & 666 & 67.96 \\
\hline 39 \& Above & 818 & 13.03 & 402 & 49.14 & 416 & 50.86 \\
\hline Total & $\mathbf{6 2 7 6}$ & $\mathbf{1 0 0}$ & $\mathbf{3 4 5 2}$ & $\mathbf{5 5}$ & $\mathbf{2 8 2 4}$ & $\mathbf{4 5}$ \\
\hline
\end{tabular}

Table 5 shows that in $19-22$ weeks, vaginal delivery occurred in $88.23 \%$ and surgery rate is $11.77 \%$ and in $23-40$ weeks it is $57.47 \%$ and $42.53 \%$ correspondingly. This decreases the maternal morbidity \& mortality.

This shows the targeted USG (18-22 weeks USG) significantly decreases the maternal morbidity and mortality by reducing the surgery rate. ${ }^{9}$

\begin{tabular}{|c|c|c|c|c|c|c|}
\hline \multicolumn{6}{|c|}{ Anomaly Group- Perinatal Mortality \& Morbidity } \\
\hline $\begin{array}{c}\text { Gestational } \\
\text { age at } \\
\text { diagnosis- } \\
\text { weeks }\end{array}$ & No. & $\%$ & \multicolumn{2}{|c|}{$\begin{array}{c}\text { Merinatal } \\
\text { Mortality }\end{array}$} & \multicolumn{2}{c|}{$\begin{array}{c}\text { Perinatal } \\
\text { Morbidity }\end{array}$} \\
\cline { 3 - 7 } & & No. & $\%$ & No. & $\%$ \\
\hline $19-22$ & 17 & 16.35 & 17 & 100 & - & - \\
\hline $23-26$ & 4 & 3.85 & 4 & 100 & - & - \\
\hline $27-30$ & 4 & 3.85 & 4 & 100 & - & - \\
\hline $31-34$ & 20 & 19.23 & 17 & 85 & 3 & 15 \\
\hline $35-38$ & 30 & 28.85 & 26 & 86.67 & 4 & 13.33 \\
\hline $39 \&$ above & 29 & 27.89 & 25 & 86.21 & 4 & 13.79 \\
\hline Total & $\mathbf{1 0 4}$ & $\mathbf{1 0 0}$ & $\mathbf{9 3}$ & $\mathbf{8 9 . 4 2}$ & $\mathbf{1 1}$ & $\mathbf{1 0 . 5 8}$ \\
\hline \multicolumn{6}{|c|}{ Table 6. Perinatal Morbidity and Mortality } \\
\hline
\end{tabular}




\begin{tabular}{|c|c|c|c|c|c|c|}
\hline \multicolumn{7}{|c|}{$\begin{array}{c}\text { Anomaly Group-Perinatal Mortality } \\
\text { \& Morbidity from 23- 40 Weeks }\end{array}$} \\
\hline \multirow{2}{*}{$\begin{array}{c}\text { Gest. } \\
\text { Age }\end{array}$} & \multirow{2}{*}{ No } & \multirow{2}{*}{$\%$} & \multicolumn{2}{c|}{$\begin{array}{c}\text { Perinatal } \\
\text { Mortality }\end{array}$} & \multicolumn{2}{c|}{$\begin{array}{c}\text { Perinatal } \\
\text { Morbidity }\end{array}$} \\
\cline { 4 - 7 } & & & No & $\%$ & No & $\%$ \\
\hline $23-40$ & 87 & 83.65 & 76 & 87.36 & 11 & 12.64 \\
\hline
\end{tabular}

\begin{tabular}{|c|c|c|c|c|c|c|}
\hline \multicolumn{2}{|c|}{ Normal Foetus Group-Perinatal Mortality \& Morbidity } \\
\hline $\begin{array}{c}\text { Gestational } \\
\text { age at } \\
\text { Diagnosis- } \\
\text { Weeks }\end{array}$ & No & $\%$ & \multicolumn{2}{|c|}{$\begin{array}{c}\text { Perinatal } \\
\text { Mortality }\end{array}$} & \multicolumn{2}{c|}{$\begin{array}{c}\text { Perinatal } \\
\text { Morbidity }\end{array}$} \\
\cline { 4 - 8 } & & & No & $\%$ & No & $\%$ \\
\hline $19-22$ & 840 & 13.38 & 10 & 1.19 & - & - \\
\hline $23-26$ & 1010 & 16.09 & 32 & 3.17 & - & - \\
\hline $27-30$ & 1216 & 19.38 & 22 & 1.81 & 34 & 2.79 \\
\hline $31-34$ & 1412 & 22.49 & 23 & 1.62 & 128 & 9.07 \\
\hline $35-38$ & 980 & 15.63 & 11 & 1.12 & 45 & 4.59 \\
\hline 39 \& above & 818 & 13.03 & 2 & 0.24 & 19 & 2.32 \\
\hline Total & $\mathbf{6 2 7 6}$ & $\mathbf{1 0 0}$ & $\mathbf{1 0 0}$ & $\mathbf{1 . 5 9}$ & $\mathbf{2 2 6}$ & $\mathbf{3 . 6 0}$ \\
\hline
\end{tabular}

In the anomaly group, perinatal morbidity rate is $105.77 \%$, perinatal mortality rate is $894.23 \%$. In the normal foetus group, in the same period the perinatal morbidity rate is $36 \%$, the perinatal mortality rate is $16 \%$, these values clearly show the perinatal morbidity and mortality is higher in the anomalous group which can be reduced by targeted USG at 18-22 weeks and decision making which in this study is with nil morbidity.

\section{DISCUSSION}

Each year, 7.9 million children are born worldwide with congenital anomalies, of which 3.3 million die. Survivors may be mentally and/or physically disabled.

The prevalence of birth defects is comparable all over the world;

1. $1.3 \%$ in the United States.

2. $2.2 \%$ in India.

3. $2 \%$ to $3 \%$ in the United Kingdom.

4. $1.4 \%$ in Tamilnadu.

5. $1.63 \%$ in MGMGH, Trichy.

1. The most prevalent conditions include congenital heart defects, orofacial clefts, Down syndrome, and neural tube defects. $5,10,11$

2. Age distribution of mothers in the study was 57 patients in 19-29 years, 30 patients in 30-39 years, and 17 patients above 39 years. Mothers were assessed for $\mathrm{Hb} \%$ and the study shows 45 mothers with $<4 \mathrm{~g} \%, 35$ mothers with $4-9 \mathrm{~g} \%$ and 24 mothers with $>9 \mathrm{~g} \%$.

3. In this study, 36 babies were male, 68 were female, sex ratio was $1: 1.9$. Birth weight of babies- 62 babies with $<2.5 \mathrm{~kg}$, and 42 babies with weight between $2.6-4 \mathrm{~kg}$.

4. Surgery was done in the newborn period for 20 babies: surgery for duodenal atresia 4 cases, VP shunting 2 cases, CDH Correction 2 cases, cleft lip repair 5 cases, cleft palate repair 2 cases, surgery for posterior urethral valve 3 cases, surgery for ileal atresia 1 case. The perinatal morbidity rate is $105.77 \%$. Mortality occurred in 15 lethal cases diagnosed at 19-22 weeks and 77 lethal cases diagnosed between 23-40 weeks and 1 case of cleft palate repair done in the postoperative period (total-93 cases) making perinatal mortality rate 894.23\%. These show the higher perinatal morbidity and mortality than the general population. ${ }^{12}$

5. Delay of delivery could have been avoided by Targeted USG. In 19-22 weeks, vaginal delivery occurred in $88.23 \%$ and surgery rate is $11.77 \%$. And in $23-40$ weeks, it is $57.47 \%$ and $42.53 \%$ correspondingly. This shows the targeted USG (18-22 weeks USG) significantly decreases the maternal morbidity and mortality by reducing the surgery rate and also the perinatal morbidity and mortality.

\section{CONCLUSION}

The results of this study show that babies admitted to a Newborn Special Care Unit had congenital abnormalities and that the commonest forms seen were mainly CVS birth defects. Based on the study the incidence of CVS congenital anomalies was found to be more common. In order to diagnose early and to reduce the perinatal mortality, we need an 18-22 weeks antenatal targeted ultrasound with complete anatomical survey.

Antenatal ultrasound is a non-invasive highly sensitive, accurate and cost-effective imaging technique which gives good results in experienced hands. Meticulous screening for pregnant ladies by ultrasound, especially in $2^{\text {nd }}$ trimester and followup of anomaly cases if required is recommended.

Public awareness about preventable risk factors like anaemia, GDM is to be created and early prenatal diagnosis and management of common anomalies is strongly recommended.

Followup USG after targeted USG, Doppler ultrasound examination, genetic karyotyping, counselling regarding the nature, prognosis, management options, recurrence rate, detailed evaluation with 3D, 4D ultrasound, MRI, counselling about termination of pregnancy, continuation of pregnancy and induction at optimum time and its benefits.9,13,14 are to be done.

The following are the recommendations to reduce Foetal and Maternal morbidity and mortality-

1. Need for targeted USG and the need for further study by foetal echo, Doppler, triple and quadruple test routinely, especially in high risk cases.

2. Need for one or more USG examinations, followup, need for high resolution USG, foetal echo, need for specialist care, (Radiologist, Obstetrician, Paediatrician, Paediatric surgeon, Neurosurgeon, Cardiologist, etc.) in decision making to reduce foetal and maternal morbidity and mortality. ${ }^{15,16}$

3. Need for counselling regarding continuation of pregnancy (non-lethal) and termination of pregnancy (lethal).

4. The limitations are due to operator expertise, quality of instrument, amount of liquor, method of termination in public health setup. 


\section{REFERENCES}

[1] Hudgins I, Cassidy SB. Congenital malformations among live births at Arvand Hospital Ahwaz, Iran. Pak J Med Sci 2008;24:33-7.

[2] Rajangam S, Devi R: Consanguinity and chromosomal abnormality in mental retardation and or multiple congenital anomalies. J Anat Soc India 2007;56(2):303.

[3] Malla BK. One year overview study of congenital anatomical malformation at birth in Maternity Hospital (Prasutigriha), Thapathali, Kathmandu. Kathmandu Univ Med J 2007;5(4):557-60.

[4] Naderi S. Congenital abnormality in newborns of consanguineous and non-consanguineous parents. J Obstet Gynecol 1979;53(2):195-9.

[5] Kumar V. Congenital abnormality of the brain. In: Kumar V, Abbas A, Fausto N. eds. Robbins and Cotran's pathological basis of disease. $7^{\text {th }}$ edn. Seattle: WB Saunders 2010:470-7.

[6] Czeizel AE, Intody Z, Modell B. What proportion of congenital abnormalities can be prevented? British Medical Journal 1993;306(6876):499-503.

[7] Turnpenny P, Ellard S. Congenital abnormalities. Emery's elements of medical genetics. Edinburgh, New York: Elsevier's-Churchill Livingstone 2005.

[8] SOGC clinical practice guideline No 200. Teratogenicity associated with preexisting and gestational diabetes. 2007.

[9] RCOG recommendations - Termination of pregnancy for foetal abnormality in England, Scotland and Wales. 2010.

[10] Stoll BG. Congenital anomalies. In: Kliegman RM, Jenson HB, Behrnan RE, et al. eds. Nelson Textbook of paediatrics. $18^{\text {th }}$ edn. Philadelphia: WB Sanders 2008:711-13. 18.
[11] Bahauddin IS, Al-Hoshan MS, Attyyaa RA, et al. Antenatal diagnosis, prevalence and outcome of major congenital anomalies in Saudi Arabia: a hospital based study. Ann Saudi Med J 2008;28(4):272-6.

[12] Ahaman A, Kidanto HL, Ngarina M, et al. Essential but not always available when needed-an interview study of Physicians experiences and views regarding use of obstetric ultrasound in Tanzania. Global Health Action 2016;9:10.

[13] McLennan A, Walker S. The Royal Australian and New Zealand College of Obstetricians and Gynaecologists recommendations-Prenatal assessment of foetal structural abnormalities. 2015;C-Obs 60:3-6.

[14] Ross AB, DeStigter KK, Coutinho A, et al. Ancillary benefits of Antenatal ultrasound an association between the introduction of a low-cost ultrasound program and an increase in the numbers of women receiving recommended antenatal treatments. BMC Pregnancy \& Childbirth 2014;14:424.

[15] Wilson JM, Jugner YG. Screening for foetal anomaliescare -National Library of Medicine-NICE guidelines updated-Time to prepare for termination of pregnancy/postnatal treatment/palliative care/infant disability/meta-analysis- serial USG with Doppler ultrasound versus selective ultrasound in pregnancy, systematic review. Nice guidelines 2008. no. 62 .

[16] Bijma HH, van der Heide A, Wildschut HL. Decision making after ultrasound diagnosis of foetal abnormality. Reproductive Health Matters 2008;16(Suppl 31):82-9. 